\title{
Outcome of Surgical Repair of Complete Rupture of Distal Biceps Tendon: A Clinical Series
}

\author{
EG Mohan Kumar, GM Yathisha Kumar* and Mohammed Noorudheen \\ Department of Orthopedic Surgery, KIMS Al Shifa Hospital, India
}

Received: October 24, 2017; Published: October 31, 2017

*Corresponding author: GM Yathisha Kumar, Department of Orthopedic Surgery, KIMS Al Shifa Hospital, India, Tel no: +91 9605648896; Email: yathishishere@gmail.com

\begin{abstract}
Complete distal biceps rupture is a rare injury as compared to proximal biceps tendon rupture. It is usually caused by an eccentric contraction of the muscle, often seen with a sudden unintentional pull or jerk. An epidemiological study showed an incidence of 1.2 ruptures per 100,000 patients per year with an average age of 47 years. It is important not to miss the diagnosis initially, since delay in surgery does affect the outcome. In low-demand patients with complete distal biceps tendon tears, non-operative treatment may be entertained provided the patient understands the potential for residual weakness, particularly of forearm supination. The surgical repair is the treatment of choice especially in high demand male patients. There are a variety of fixation methods including bone anchors, suspension techniques, bone tunnels with interference screws, and transosseus sutures. No one technique has emerged as the gold standard and the choice remains that of surgeon. In this case series we present couple of patients with distal biceps tendon rupture one with acute rupture and another with chronic rupture treated surgically. We conclude patients do benefit from surgical repair.
\end{abstract}

Keywords: Distal biceps tendon; Acute; Chronic; Tendon tear; Elbow; Repair

Abbreviations: USG: Ultra Sono Gram; MRI: Magnetic Resonance imaging ; ASES: American Shoulder and Elbow Surgeons; LABC: Lateral Ante Brachial Cutaneous; PREE: Patient-Rated Elbow Evaluation

\section{Introduction}

Nearly $90 \%$ of biceps tendon ruptures occur in the proximal biceps and involve the long head of biceps. The remaining ruptures occur in the distal biceps tendon representing only $3 \%$ of biceps muscle injuries. The mechanism of injury in distal rupture involves a strong eccentric contraction of the biceps tendon against unanticipated resistance. The injury is most common in the dominant arm of middle-aged men. Symptoms include weakness in elbow flexion (by 30\%) and forearm supination (by 40\%) [1].

Some individuals may maintain reasonable function after non-operative treatment of a ruptured distal biceps tendon, biomechanical and clinical studies suggest that most individuals benefit from surgical repair or reconstruction [2]. The surgical treatment of distal biceps tendon tears has been studied extensively. The techniques available for repair involve a three-level distinction: anatomic versus non-anatomic repair, single-incision versus double-incision exposure and fixation method (most commonly the use of cortical button, interference screws, transosseous sutures or suture anchors) [2,3].

The number of reported distal biceps tendon tears seems to have increased over the last few years, likely related to better understanding and improved diagnostic methods [4] However in a number of instances, the diagnosis is initially missed. In this case series we present couple of patients with complete distal biceps tendon rupture; one with acute rupture and another with chronic rupture. We discuss the diagnosis, treatment, outcome and complications of surgical repair of the tendon using suture anchor.

\section{Clinical Series}

\section{Case 1}

63 year old left dominant handed male patient sustained injury to left elbow at work site one month before he presented to us,while he was trying to cut a banana tree it was about to fall on his feet, while he tried to catch the tree before it fell. He had a painful 'pop' at the time of injury. He noticed painful range of movement of left elbow and difficulty in using left elbow. Initially he consulted an orthopaedic surgeon and was managed with a sling and analgesics, when he presented to us he had developed painful restriction of range of movement and was so unhappy about the initial treatment. On inspection there was flattening of the distal contour of the arm as compared to opposite arm (Figure 1), mild tenderness at anticubital fossa. He had weakness of flexion and supination. 


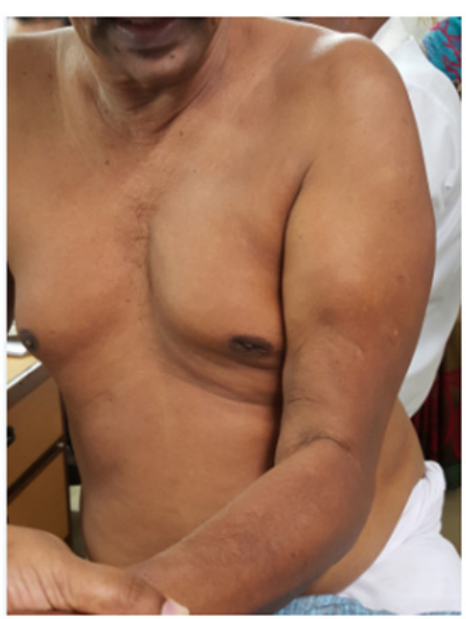

Figure 1: At presentation note the flattening of the distal contour of the arm.

On 'hook test' (Figure 2) we could not hook finger around any anterior structures with elbow in flexed and supinated position. Pre operative quick DASH score was 57. X-ray showed no bony abnormality and an MRI confirmed complete rupture (Figure 3 ) and marked retraction of distal biceps tendon from bicipital tuberosity of the radius. Since the duration of injury was just one month we planned for reinsertion of tendon to bicipital tuberosity using fibre wire and an anchor screw. After pre anaesthesia evaluation patient was taken up for surgery. We went through anterior approach using a curved single incision over antecubital fossa without a tourniquet, and retrieved the tendon from the superior part of the incision (Figure 4).

a)
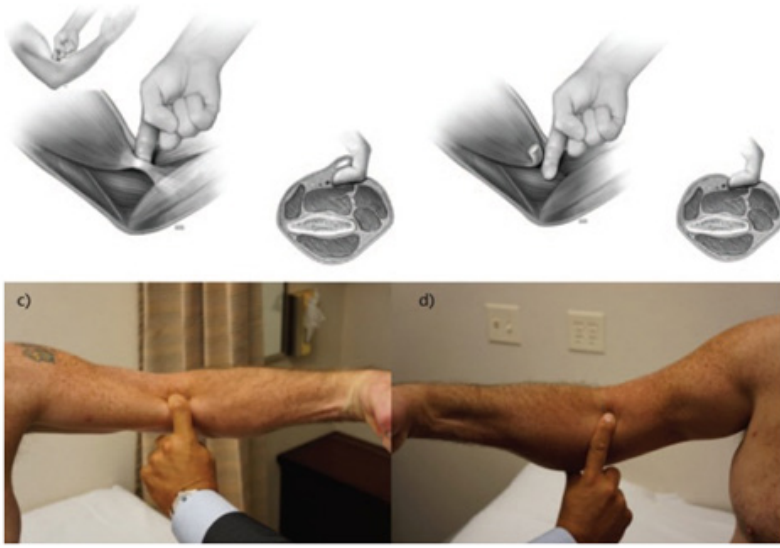

Figure 2: The hook test for distal biceps tendon, as described by O'Driscoll [6].

a. The normal test in which the examiner's fingers can be hooked under the biceps tendon.

b. The abnormal test, in which the examiner is unable to hook the distal biceps tendon.

c. Demonstration of a normal hook test. As shown, a cord-like structure is felt under the index finger.

d. Clinical picture demonstrating an abnormal hook test. The examiner is unable to feel the cord-like structure corresponding to the distal biceps tendon.

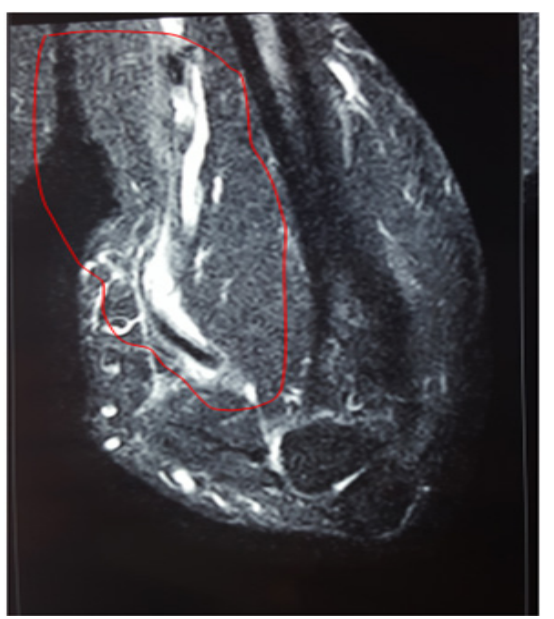

Figure 3: MRI showing complete rupture and marked retraction of distal beceps tendon from bicipital tuberosity of radius.

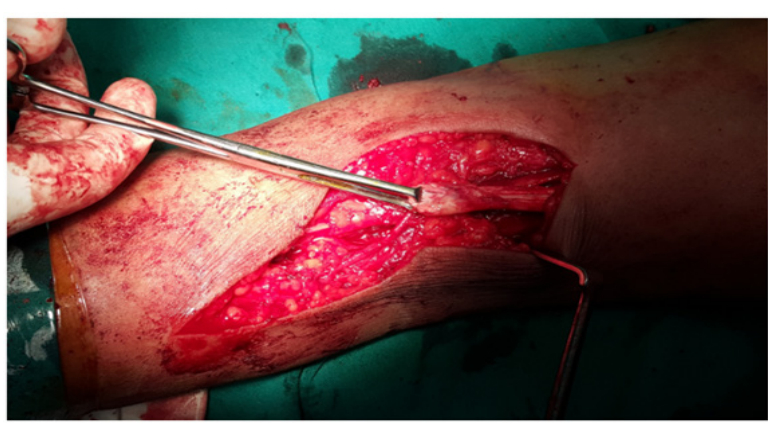

Figure 4: Retrieval of the ruptured tendon from the proximal part of incision.

On the distal part of the incision further dissection was done and recurrent branch of radial artery was ligated (Figure 5) to avoid injury to it. Bicepital tuberosity on radius was identified and bed prepared (Figure 6). An anchor screw was passed into it and with the fibre wire, tendon was re attached (Figure 7), as we expected there was enormous tension on tendon and hence a long arm slab in 1100 of flexion and supination was given. Patient was discharged on second post op day and at one month follow up slab and sutures were removed, elbow mobilisation started (Figure 8). Wound was healed well and at 2 months post of he had near normal range of movement and without any pain and quick DASH score improved to 11 at 2 months post op and it is improving further.

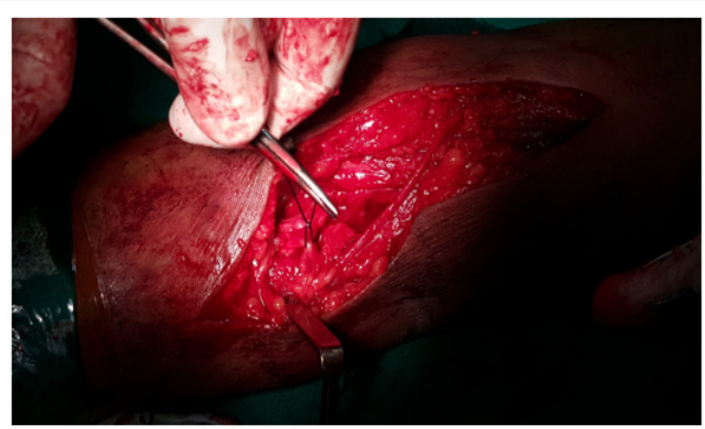

Figure 5: Ligation of recurrent branch of radial artery. 


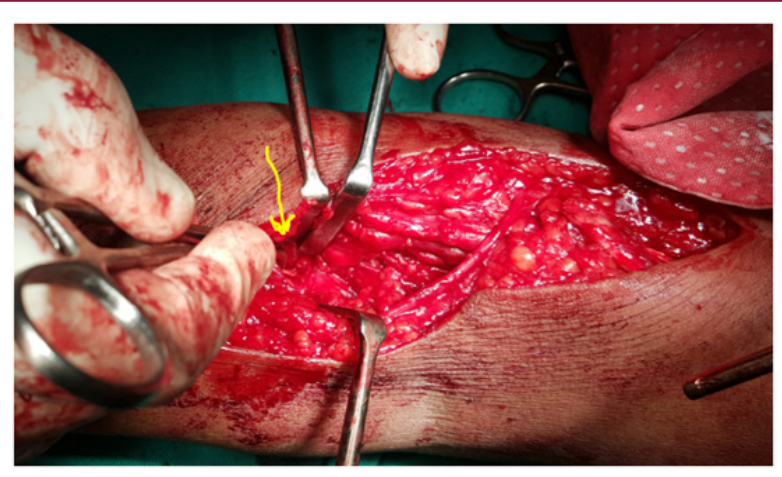

Figure 6: Bicepital tuberosity on radius.

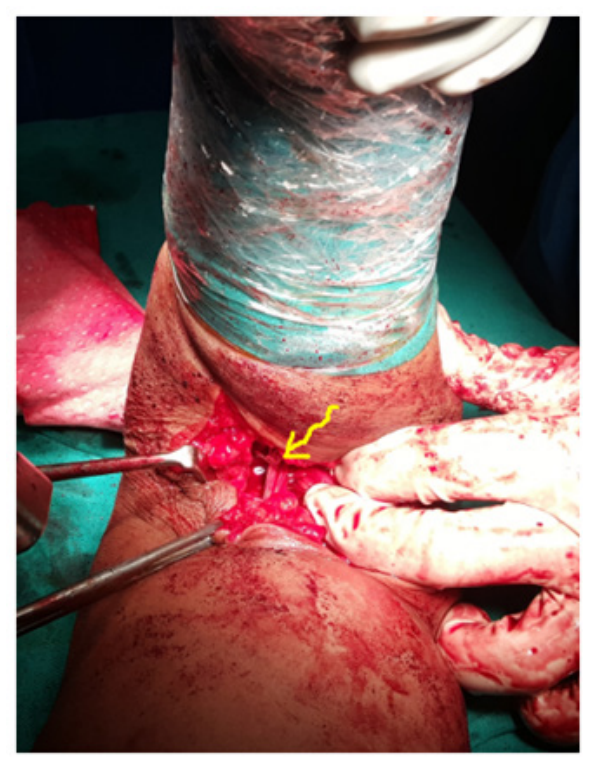

Figure 7: Reattachment of tendon using suture anchor and fibre wire.

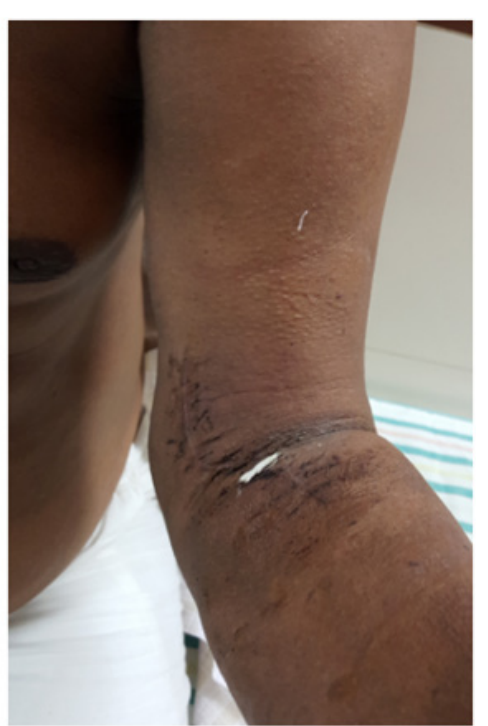

Figure 8: At 1 month follow up POP slab was removed and sutures removed wound healed well.

\section{Case 2}

A 44 year old right hand dominant male farmer patient sustained distal bicepital tendon rupture following a fall from tree while he was trying to cut a branch of tree and he lost control and fell down and tried to catch another branch of the tree to save himself and he heard a painful pop at that time and finally he fell on the ground. Fortunately he did not have any major fractures but noticed pain and swelling around the elbow. On the same day he presented to us. On examination swelling and ecchymosis in the distal arm and proximal forearm. Severe tenderness was noted. X ray of right elbow was normal and an MRI showed complete tear of distal biceps tendon. He was treated similarly as described in case one and biceps was reattached using fibre wire and suture anchor. Post operative protocol was also similar as case one and at 3 months follows up his quick DASH score improved to zero and he returned back to work.

\section{Discussion}

Distal biceps rupture is a rare injury as compared to proximal biceps tendon rupture usually caused by an eccentric contraction of the muscle, often seen with a sudden unintentional pull or jerk. An epidemiological study showed an incidence of 1.2 ruptures per 100,000 patients per year with an average age of 47 years [5]. Unlike proximal biceps tendon rupture which leave only a cosmetic deformity (with little or no functional disability), the distal biceps tendon injury affects functional outcome of elbow resulting in stiffness, chronic pain, weakness of flexion and supination. Unfortunately, the implications of this injury are high, in demanding male labourers. In a number of instances, the diagnosis is initially missed. In such situations patients are extremely unhappy. As seen in our patient (case one) his diagnosis was missed initially and when he presented to us at one month post injury he was very unhappy about initial treatment and outcome.

The diagnosis of complete distal biceps tendon tears can be established based on patient history and physical examination. $\mathrm{X}$ ray elbow may be normal; Ultra sonogram (USG) and magnetic resonance imaging (MRI) provide more valuable information. Patients may report a painful 'pop' at the time of injury. A useful clinical test was described by $0^{\prime}$ Driscoll et al. [6,7], the so-called 'hook test'. The patient is asked to look at the palm of his hand on the affected side with the shoulder elevated, the elbow flexed at $90^{\circ}$ and the forearm in supination. An intact distal biceps tendon allows the examiner to hook his fingers around the cord-like structure. If the bicep is torn, since the distal brachial is is flat, the examiner will not be able to hook his finger around any anterior structures. This test was elicited in patient with chronic distal biceps tendon rupture (case 1) We did not try this test in other patient with acute tendon rupture because it was painful (Figure 2).

Nonoperative treatment in symptomatic patients has been shown to result in a $30-50 \%$ loss in supination strength and $20 \%$ loss in elbow flexion strength [8]. Thus the surgical repair is the treatment of choice especially in high demand male patients. So, the consensus of opinion is that acute ruptures should be repaired primarily if possible, and there are a variety of fixation methods including bone anchors, suspension techniques, bone tunnels with interference screws, and transosseus sutures [9]. No one technique has emerged as the gold standard and the choice remains that of surgeon. 
The techniques available for repair involve a three-level distinction: anatomic versus non-anatomic repair, single-incision versus double-incision exposure and various fixation methods. Randomised controlled trial was conducted to compare acute DBT tears treated surgically with a single-incision technique (fixation with two suture anchors) or double-incision technique (fixation with transosseous bone tunnels) [10]. The authors found that both techniques provided similar results in terms of pain, American Shoulder and Elbow Surgeons (ASES) elbow scores, and functional sub-scores, DASH score, patient-rated elbow evaluation (PREE) score, and isometric extension, pronation or supination strength.

However, the double-incision technique resulted in significantly higher strength for elbow flexion when compared to the singleincision technique (104\% Vs $94 \%$, respectively). We have used single incision technique in both cases and used anchor screw for fixation. In acute cases this approach gives excellent exposure for retrieval, preparation and fixation at radial tuberosity. In chronic cases if there is associated lacertus fibrosus tear, tendon retracts so much and generates lot of tension when it is brought down and hence it requires immobilisation in flexion and supination. In situation where severe retraction of tendon requires reconstruction this is best done with auto graft. Outcome of our patients is excellent. Quick DASH score improved from 57 to 11 at 2 months post op and it is improving further in patient with chronic rupture and quick DASH score improved to 0 at 3 months post-op in patient with acute rupture (Figures $9 \& 10$ ).

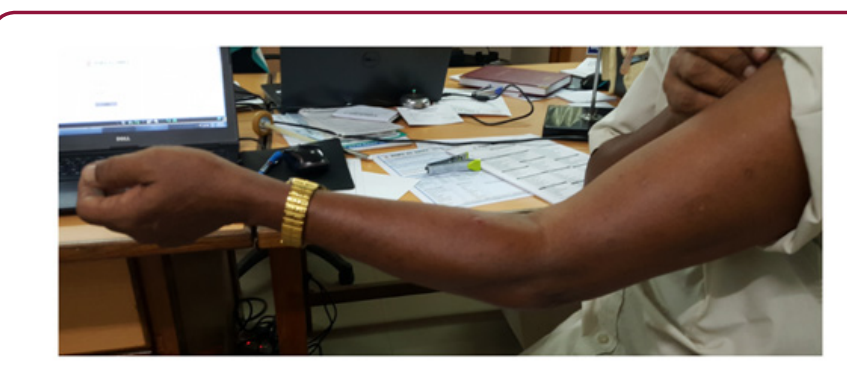

Figure 9: At 2 months follow up.

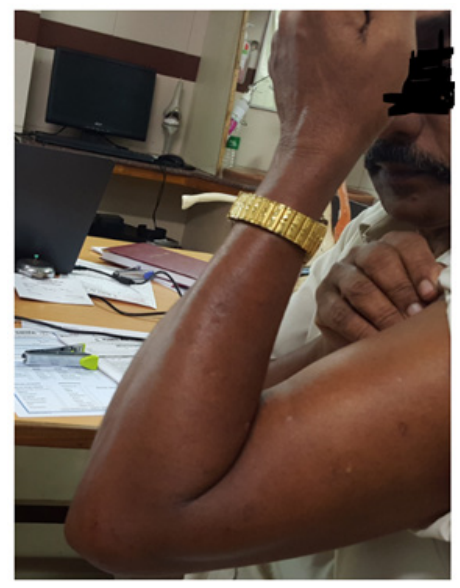

Figure 10: At 2 months follow up.
Injuries to posterior interosseous nerve and the lateral ante brachial cutaneous (LABC) nerve have been reported in 5\% to $40 \%$ of elbows respectively, more commonly with a single anterior incision [10,11]. Heterotrophic ossification may be seen on radiographs after distal biceps tendon repair using any exposure or fixation technique, but it seems to be more common and tends to interfere more with forearm rotation using a two-incision technique. Care should be taken not to expose the ulna to prevent cross union [3]. We don't have such complication in either case.

\section{Conclusion}

Distal biceps tendon rupture is a relatively rare injury usually caused by an eccentric contraction of the muscle in middle aged men. It is common on the dominant side. It is important not to miss the diagnosis initially, since delay in surgery does affect the outcome. In low-demand individuals acute complete tears are occasionally treated non-operatively, but most patients benefit from surgical repair.

\section{Acknowledgement}

Sincere acknowledgement to Dr Ajay Kumar, all PG students, Sanith, Manikandan, Faizal Babu, Sreejith for making substantial contributions to this study.

\section{References}

1. Ioannis Sarris, Dean G Sotereanos (2002) Distal biceps tendon ruptures; Journal of the American Society for Surgery of the Hand 2(3 ): 121-128.

2. Miyamoto RG, Elser F, Millett PJ (2010) Distal biceps tendon injuries. J Bone Joint Surg Am 92(11): 2128-2138.

3. Sutton KM, Dodds SD, Ahmad CS, Sethi PM. Surgical treatment of distal biceps rupture. J Am Acad Orthop Surg 18(3): 139-148.

4. Kelly MP, Perkinson SG, Ablove RH, Tueting JL (2015) Distal Biceps Tendon Ruptures: An Epidemiological Analysis Using a Large Population Database. J Sports Med 3(8): 2012-2017.

5. Safran MR, Graham SM (2012) Distal biceps tendon ruptures: incidence, demographics, and the effect of smoking. Clin Orthop Relat Res (404): 275-283.

6. O'Driscoll SW, Goncalves LB, Dietz P (2007) The hook test for distal biceps tendon avulsion. Am J Sports Med 35(11): 1865-1869.

7. Alentorn Geli, Eduard, Andrew T Assenmacher, Joaquín Sánchez-Sotelo (2016) Distal Biceps Tendon Injuries: A Clinically Relevant Current Concepts Review. EFORT Open Reviews 1(9): 316-324.

8. Baker BE, Bierwagen D (1985) Rupture of the distal tendon of the biceps brachii. Operative versus non-operative treatment. J Bone Joint Surg Am 67(3): 414-417.

9. Miyamoto RG, Elser F, Millett PJ (2010) Distal biceps tendon injuries. Bone Joint Surg Am 92(11): 2128-2138.

10. Grewal R, Athwal GS, MacDermid JC, Faber KJ, Drosdowech DS et al. (2012) Single versus double-incision technique for the repair of acute distal biceps tendon ruptures: a randomized clinical trial. J Bone Joint Surg Am 9(13): 1166-1174.

11. Morrey BF (2015) Repair of distal biceps tendon rupture. In: Morrey BF (Eds.), edn. Master's technique in orthopaedic surgery.PA: Wolters Kluwe, The elbow Philadelphia, USA, pp. 267-289. 


\begin{tabular}{ll} 
BIOMEDICAL & Assets of Publishing with us \\
RESEARCHES & - Global archiving of articles \\
\hline
\end{tabular}

